\title{
Robot-assisted stereoelectroencephalography exploration of the limbic thalamus in human focal epilepsy: implantation technique and complications in the first 24 patients
}

\author{
*Ganne Chaitanya, MBBS, PhD, ${ }^{1,2}$ Andrew K. Romeo, MD, ${ }^{3}$ Adeel Ilyas, MD, ${ }^{2,3}$ Auriana Irannejad,,${ }^{1,2}$ \\ Emilia Toth, PhD, ${ }^{1,2}$ Galal Elsayed, MD, ${ }^{3}$ J. Nicole Bentley, MD, ${ }^{3}$ Kristen O. Riley, MD, ${ }^{3}$ and \\ Sandipan Pati, MD1,2
}

1Department of Neurology, ${ }^{2}$ Epilepsy and Cognitive Neurophysiology Laboratory, and ${ }^{3}$ Department of Neurosurgery, University of Alabama at Birmingham, Alabama

OBJECTIVE Despite numerous imaging studies highlighting the importance of the thalamus in a patient's surgical prognosis, human electrophysiological studies involving the limbic thalamic nuclei are limited. The objective of this study was to evaluate the safety and accuracy of robot-assisted stereotactic electrode placement in the limbic thalamic nuclei of patients with suspected temporal lobe epilepsy (TLE).

METHODS After providing informed consent, 24 adults with drug-resistant, suspected TLE undergoing evaluation with stereoelectroencephalography (SEEG) were enrolled in the prospective study. The trajectory of one electrode planned for clinical sampling of the operculoinsular cortex was modified to extend it to the thalamus, thereby preventing the need for additional electrode placement for research. The anterior nucleus of the thalamus (ANT) $(n=13)$ and the medial group of thalamic nuclei (MED) $(n=11)$, including the mediodorsal and centromedian nuclei, were targeted. The postimplantation CT scan was coregistered to the preoperative MR image, and Morel's thalamic atlas was used to confirm the accuracy of implantation.

RESULTS Ten (77\%) of 13 patients in the ANT group and 10 (91\%) of 11 patients in the MED group had electrodes accurately placed in the thalamic nuclei. None of the patients had a thalamic hemorrhage. However, trace asymptomatic hemorrhages at the cortical-level entry site were noted in $20.8 \%$ of patients, who did not require additional surgical intervention. SEEG data from all the patients were interpretable and analyzable. The trajectories for the ANT implant differed slightly from those of the MED group at the entry point-i.e., the precentral gyrus in the former and the postcentral gyrus in the latter.

CONCLUSIONS Using judiciously planned robot-assisted SEEG, the authors demonstrate the safety of electrophysiological sampling from various thalamic nuclei for research recordings, presenting a technique that avoids implanting additional depth electrodes or compromising clinical care. With these results, we propose that if patients are fully informed of the risks involved, there are potential benefits of gaining mechanistic insights to seizure genesis, which may help to develop neuromodulation therapies.

https://thejns.org/doi/abs/10.3171/2020.1.FOCUS19887

KEYWORDS temporal lobe epilepsy; thalamus; stereotactic electroencephalography; anterior nucleus of the thalamus; mediodorsal nucleus; centromedian nucleus

ABBREVIATIONS ANT = anterior nucleus of the thalamus; $\mathrm{CeM}=$ centromedian nucleus of the thalamus; $\mathrm{DBS}=$ deep brain stimulation; $\mathrm{EZ}=$ epileptogenic zone; $\mathrm{MED}=$ medial group of thalamic nuclei; SEEG = stereoelectroencephalography; TLE = temporal lobe epilepsy.

ACCOMPANYING EDITORIAL DOI: 10.3171/2020.1.FOCUS2069.

SUBMITTED November 17, 2019. ACCEPTED January 24, 2020.

INCLUDE WHEN CITING DOI: 10.3171/2020.1.FOCUS19887.

${ }^{*}$ G.C., A.K.R., and A. llyas contributed equally to this work. 
$\mathrm{A}$ CCURATE localization of the epileptogenic zone (EZ) in temporal lobe epilepsy (TLE) is paramount to optimizing outcomes following resection or ablation. ${ }^{44}$ However, despite significant technological advancements in imaging and surgical tools, seizurefreedom outcomes have plateaued at $45 \%-65 \%$ over the last decade, necessitating a continued investigation of how distributed brain regions interact to cause seizures..$^{39}$ Growing evidence suggests that networks of functionally and structurally connected areas, both within and outside of mesial temporal structures, contribute to EZs. ${ }^{4,10}$, 19,32 Among extratemporal structures implicated in seizure inception, increased thalamotemporal structural and functional connectivity independently predicts poor surgical outcomes..$^{17}$ Experimental studies in preclinical models of limbic epilepsy support the hypothesis that the thalamus can regulate limbic seizures, and the stage at which ictogenesis is regulated (i.e., initiation, propagation, or termination) depends on the functional connectivity of the thalamic nuclei with limbic structures. ${ }^{6,9,36}$ Furthermore, modulation of the "limbic" thalamic nuclei (i.e., the anterior, midline and mediodorsal, and intralaminar centromedian $[\mathrm{CeM}]$ nuclei) through chemical, optogenetic, or electrical perturbation can interrupt focal seizures. ${ }^{6,16,25,37}$ However, despite numerous preclinical and imaging studies highlighting the importance of the thalamus in surgical prognosis,${ }^{17}$ there are very limited human electrophysiological studies targeting the limbic thalamus.

Over the last decade, clinicians have implanted subdural grids, hybrid macro-micro depth electrodes, and laminar electrodes for high-density intracranial recordings from both the superficial and deep cortexes. ${ }^{41,43}$ Although the thalamus is likely a rich source of data about seizure regulation, propagation, and sleep dysfunction, ${ }^{12,15}$ progress in this area is relatively slow due to ethical and safety concerns. However, future therapeutic developments may be highly informed by understanding thalamotemporal causal interactions during seizures. Here, we present the technical nuances, safety details, and accuracy data from stereoelectroencephalography (SEEG) implantation of electrodes into the limbic thalamus during the presurgical evaluation of patients with suspected TLE.

\section{Methods}

\section{Patient Selection}

Enrolled patients included those deemed eligible for SEEG after consensus recommendation from a multidisciplinary epilepsy conference consisting of neurologists, neurosurgeons, neuropsychologists, and nurses. Adults with drug-resistant, suspected TLE undergoing SEEG evaluation were eligible to participate in the study. Surgeons modified the trajectory of one electrode planned for clinical sampling to extend to the thalamus, obviating the need to implant an additional electrode for thalamic sampling. A 5-stage evaluation process helped streamline the process of recruiting considerably homogeneous groups of patients with suspected TLE (mesial and/or temporal plus) who would receive the thalamic implant (Fig. 1). The investigators approached the potential candidates for thalamic SEEG at the outpatient follow-up clinic visit, and written informed consent was obtained in accordance with protocols approved by the University of Alabama at Birmingham Institutional Review Board.

\section{Thalamic Trajectory}

In this study, the limbic thalamic nuclei that were targeted were as follows: 1) anterior nucleus of the thalamus (ANT), including anterior ventral, anterior dorsal, and anterior medial subnuclei; and 2) medial group of thalamic nuclei (MED), including mediodorsal and CeM subnuclei. Neurosurgeons planned trajectories using T1-weighted

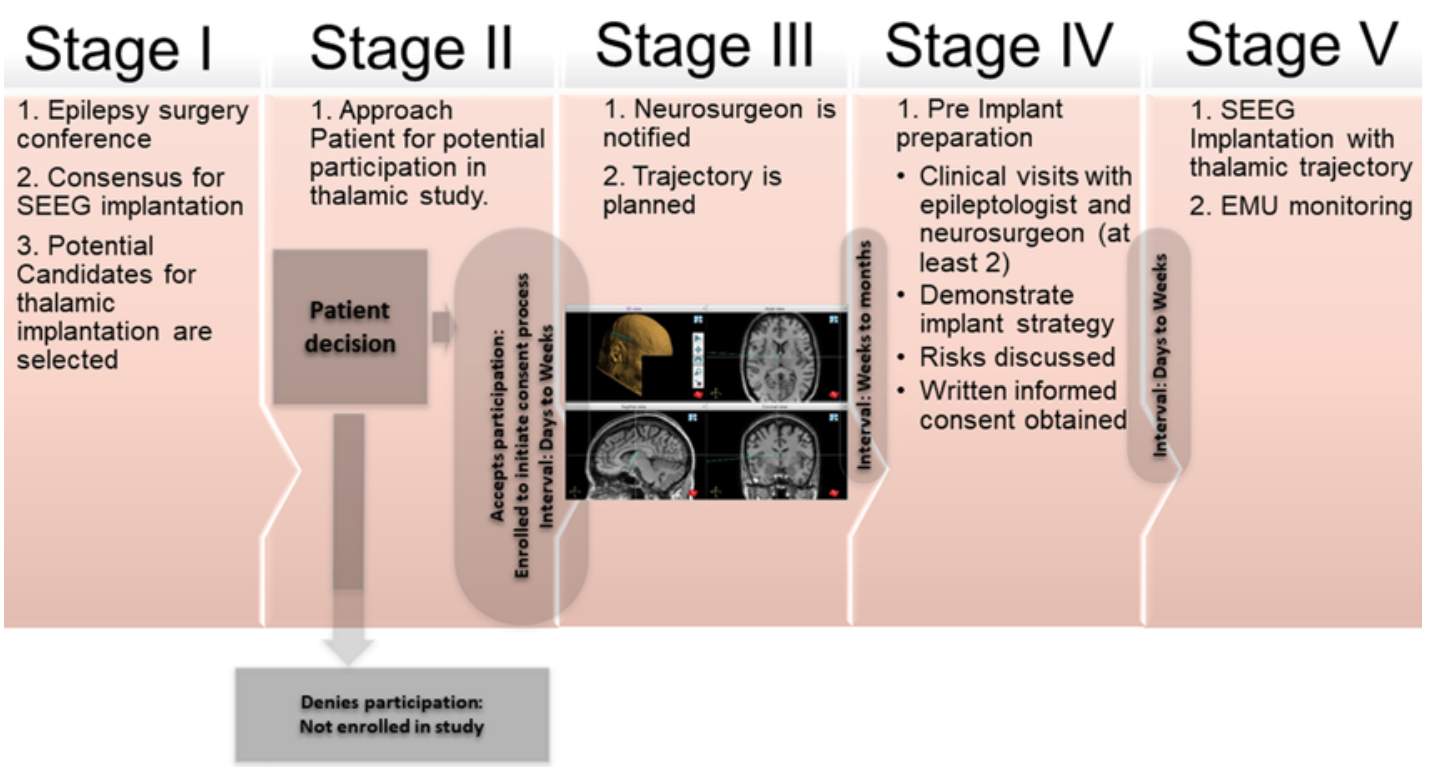

FIG. 1. Patient selection and SEEG implantation: a multistage process to obtain informed consent from eligible patients scheduled to undergo SEEG investigation for localization of epilepsy. EMU = epilepsy monitoring unit. 
MRI sequences with gadolinium contrast using robotic stereotactic planning software (ROSA; Medtech). MRI scans were acquired using the epilepsy protocol in a Philips Achieva 1.5T scanner (matrix size $384 \times 384 \mathrm{~mm} / 32 \times$ $432 \mathrm{~mm}$, slice thickness $1.2 \mathrm{~mm}$, TR $7 \mathrm{msec}$, TE $3 \mathrm{msec}$, interslice gap $1.2 \mathrm{~mm}$, flip angle $8^{\circ}$ ), Philips Achieva 3T scanner (matrix size $256 \times 256 \mathrm{~mm} / 528 \times 528 \mathrm{~mm}$, slice thickness $1 \mathrm{~mm} / 1.2 \mathrm{~mm}$, TR $6 \mathrm{msec}$, TE $3 \mathrm{msec} / 4 \mathrm{msec}$, interslice gap $1 \mathrm{~mm} / 1.2 \mathrm{~mm}$, flip angle $8^{\circ} / 9^{\circ}$ ), Philips Ingenia 1.5T scanner (matrix size $512 \times 512 \mathrm{~mm} / 432 \times 432$ $\mathrm{mm}$, slice thickness $1 \mathrm{~mm}$, TR $7 \mathrm{msec}$, TE $3 \mathrm{msec}$, interslice gap $1 \mathrm{~mm}$, flip angle $8^{\circ}$ ), Philips Ingenia 3T scanner (matrix size $528 \times 528 \mathrm{~mm} / 432 \times 432 \mathrm{~mm}$, slice thickness $1.2 \mathrm{~mm}$, TR $7 \mathrm{msec}$, TE $3 \mathrm{msec}$, interslice gap $1.2 \mathrm{~mm}$, flip angle $9^{\circ}$ ), or SIEMENS Skyra 3T scanner (matrix size 256 $\times 256 \mathrm{~mm}$, slice thickness $0.9 \mathrm{~mm}$, TR $7 \mathrm{msec}$, TE $3 \mathrm{msec}$, interslice gap $0 \mathrm{~mm}$, flip angle $8^{\circ}$ ).

Based on a visual reference to Morel's thalamic atlas, the nuclei were determined to be located in relation to the following landmarks ${ }^{21}$ while planning the trajectory. The ANT was identified by its close relationship to the foramen of Monro and the venous angle formed by the confluence of thalamostriate and septal veins lying immediately posterior and lateral (Fig. 2). Extending a trajectory that incorporated the frontal operculum and insula, the thalamic region of interest was targeted without requiring an additional electrode. For the MED group of thalamic nuclei, neurosurgeons targeted the ventral midline thalamus near the massa intermedia, providing recordings from the centromedial or mediodorsal nuclei. The broadest midline nuclear segment was anterior and ventral to the massa intermedia, where the reuniens and central median nuclei are located. ${ }^{33}$ The massa intermedia could be visualized in 5 of the 8 MED thalamic groups of patients. When not distinct, the anteroposterior midpoint at the level of the anterior commissure-posterior commissure plane was chosen. $^{5}$ The inferior and nearly parallel relationship with the ANT provided further anatomical confirmation.

Following SEEG implantation, patients were initially monitored in the neurosurgical ICU for 24 hours. Highresolution postimplantation head CT scans were obtained within 24 hours. Subsequently, the patients were transferred to the epilepsy monitoring unit for seizure localization and mapping.

\section{Measuring Accuracy}

Postimplantation CT scans were acquired using Philips Brilliance64 and Brilliance16P scanners (matrix size $512 \times 512 \mathrm{~mm}$, slice thickness $1 \mathrm{~mm}, 120 \mathrm{kVp}$ [peak kilovoltage], interslice gap $1 \mathrm{~mm}$, exposure time $1550 \mathrm{sec} / 727$ sec). The CT scans were coregistered to the preoperative MR images using Advanced Normalization Tools (Fig. 3). ${ }^{3}$ Electrodes were localized using Lead-DBS software (www.lead-dbs.org), and trajectories were mapped using iElectrodes software. ${ }^{7,18}$ Coregistered images were normalized to ICBM152-2009b nonlinear asymmetrical atlases using nonlinear diffeomorphic normalization algorithms, and brain shift corrections were performed using Schönecker normalizations, providing refined registration of subcortical structures. ${ }^{35}$ Registrations were checked manually for errors in 3D Slicer. The data were
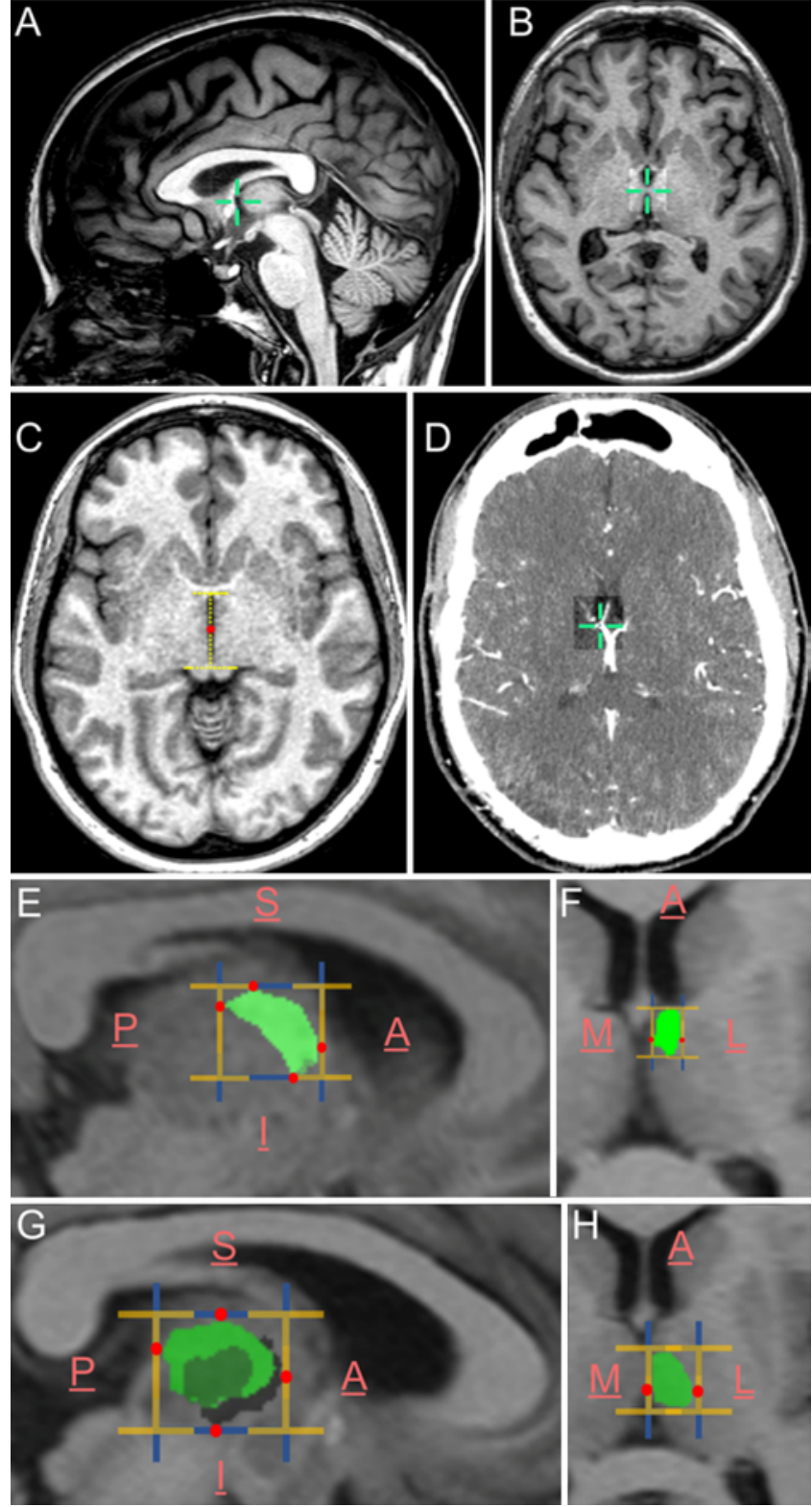

FIG. 2. Landmarks for targeting the thalamic nuclei. The foramen of Monro as the anteromedial extent $(\mathbf{A})$, the massa intermedia as the medial extent (B), the midpoint (red dot) of the anteroposterior length (yellow line) of the third ventricle in axial view as the inferior extent (C) (measured at the level of the superior colliculus and the venous angle as the anteromedial extent), and the confluence of the anterior septal vein, thalamostriate vein, and internal cerebral vein $(D)$ were the major landmarks used while targeting the electrodes. Green crosshairs in A, B, and $D$ indicate the coordinates of the aforementioned landmarks. $E$ and F: The probable extents of ANT as per Morel's atlas. G and H: The probable extents of MED (CeM + mediodorsal subnuclei) as per Morel's atlas. The yellow brackets indicate the anteroposterior extent of nuclei in $\mathrm{E}$ and $\mathrm{G}$ and the medial to lateral extent in $\mathrm{F}$ and $\mathrm{H}$. The red dots indicate the farthest extent of the thalamic nucleus in any given direction. The blue brackets indicate the superoinferior extent of the nuclei. $A=$ anterior; I = inferior; $L=$ lateral; $M=$ medial; $P=$ posterior; $S=$ superior. 

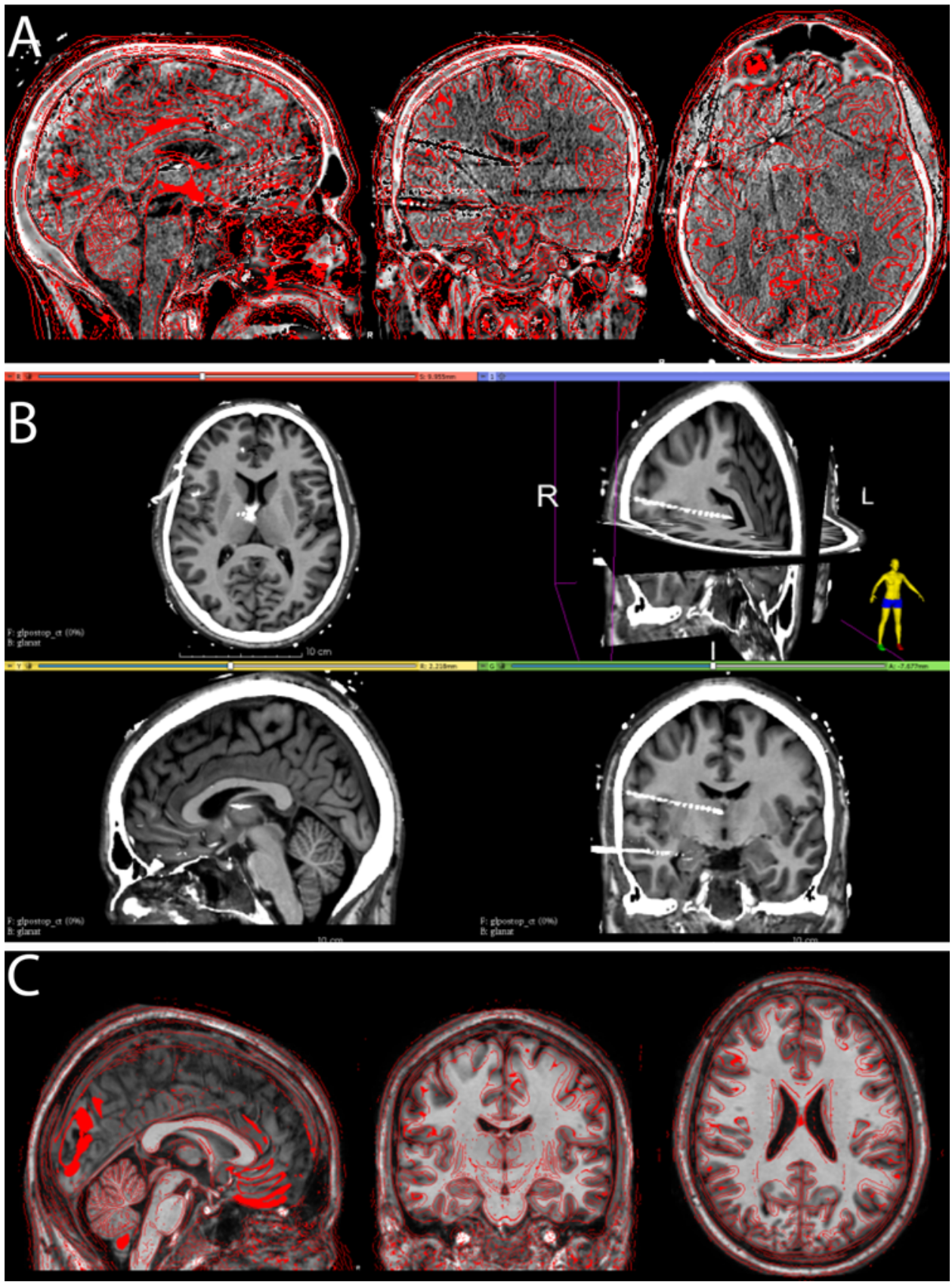

FIG. 3. Coregistration strategy. A: The postimplantation CT scan was coregistered onto the preoperative MR image to determine the postoperative target of the thalamic targets using Advanced Normalization Tools in Lead-DBS. B: Following coregistration, a manual verification of the anatomical landmarks and the overlap of the inner and outer tables of the MR image and CT scan were used to ensure accurate coregistration. In particular, the most probable location of the thalamic electrode was confirmed. C: The coregistered MR image and CT scan were then normalized to the MNI (Montreal Neurological Institute) space. The coregistered images were then resolved for finer registration and brain shifts using the Schönecker normalization algorithm to improve the registration of the subcortical structure (3D Slicer).

then visualized using Morel's atlas..$^{21}$ To map the trajectories, the coregistered and normalized CT and MRI scans were imported along with the corresponding CT mask into iElectrodes and registered with the AAL2 atlas to identify their cortical-subcortical locations. ${ }^{28,40}$ As a final step in establishing the accuracy of the implant strategy from our experience, we estimated the postimplantation accurate location of the thalamic target as well as the linear component distances along the $\mathrm{x}, \mathrm{y}$, and $\mathrm{z}$ axes and the Euclidean distances from the landmarks. Postexplantation CT scans were also obtained to look for postoperative hemorrhage or edema. If found, hemorrhage was graded using McGovern's SEEG hemorrhage grading system..$^{23}$ 
TABLE 1. Clinical presentation of the 24 participants and summary of post-SEEG localization of the seizure pathology and the treatment strategy

\begin{tabular}{|c|c|c|c|c|c|c|c|c|c|c|}
\hline $\begin{array}{c}\text { Thalamic } \\
\text { Nucleus \& } \\
\text { Participant No. }\end{array}$ & Sex & $\begin{array}{l}\text { Age } \\
\text { (yrs) }\end{array}$ & $\begin{array}{c}\text { Age at } \\
\text { Epilepsy } \\
\text { Onset (yrs) }\end{array}$ & $\begin{array}{l}\text { Duration } \\
\text { of Epilepsy } \\
\text { (yrs) }\end{array}$ & $\begin{array}{l}\text { No. of } \\
\text { Failed } \\
\text { AEDs }\end{array}$ & $\begin{array}{l}\text { Preimplant } \\
\text { MRI }\end{array}$ & $\begin{array}{c}\text { No. of } \\
\text { Electrodes }\end{array}$ & $\begin{array}{l}\text { Laterality } \\
\text { of Thalamic } \\
\text { Electrode }\end{array}$ & Post-SEEG EZ & Treatment \\
\hline \multicolumn{11}{|l|}{ ANT } \\
\hline 1 & $\mathrm{~F}$ & 42 & 38 & 3.5 & 5 & HS+ & 9 & $\mathrm{Lt}$ & Lt TLE subtype* & $\begin{array}{l}\text { Pt deferred surgical } \\
\text { therapy }\end{array}$ \\
\hline 2 & $M$ & 24 & 6 & 18.5 & 2 & $\mathrm{~N}$ & 14 & $\mathrm{Rt}$ & Rt MTLE & Rt ATL \\
\hline 3 & $\mathrm{~F}$ & 47 & 41 & 6.2 & 7 & $\mathrm{~N}$ & 14 & Rt & Bilat TLE & Bilat HG RNS \\
\hline 4 & $\mathrm{M}$ & 37 & 29 & 8.0 & 4 & $\mathrm{EH}$ & 10 & Rt & Rt TLE plus† & Rt HG \& OrbF RNS \\
\hline 5 & $\mathrm{~F}$ & 57 & 53 & 4.2 & 9 & $\mathrm{~N}$ & 13 & Rt & Rt TLE: Tp subtype & Rt ATL \\
\hline 6 & $\mathrm{~F}$ & 51 & 40 & 11.0 & 3 & $\mathrm{~N}$ & 11 & Rt & Rt TLE plus $†$ & $\begin{array}{l}\text { Rt ATL extended to Ins } \\
\quad \& \text { OperG }\end{array}$ \\
\hline 7 & $F$ & 59 & 16 & 43.2 & 10 & $\mathrm{EH}$ & 14 & Rt & $\begin{array}{l}\text { Multifocal: rt TLE, } \\
\text { parietal }\end{array}$ & Palliative rt HG LiTT \\
\hline 8 & $M$ & 34 & 10 & 23.9 & 11 & $\mathrm{EH}$ & 20 & Rt & $\begin{array}{l}\text { Multifocal: rt TLE, } \\
\text { frontal }\end{array}$ & Palliative rt ATL \\
\hline 9 & M & 46 & 6 & 39.9 & 4 & HS+ & 15 & Rt & Rt MTLE & Rt ATL \\
\hline 10 & $\mathrm{~F}$ & 36 & 31 & 4.5 & 6 & $\mathrm{~N}$ & 16 & $\mathrm{Lt}$ & Lt TLE plus, $†$ rt MTLE & Bilat HG RNS \\
\hline 11 & $M$ & 29 & 8 & 20.6 & 4 & $\mathrm{~N}$ & 12 & $\mathrm{Lt}$ & Lt TLE plus† & $\begin{array}{l}\text { Mesial OrbF, ACingG } \\
\text { resected, planned } \\
\text { HG RNS }\end{array}$ \\
\hline 12 & $\mathrm{~F}$ & 48 & 41 & 7.5 & 5 & HS+ & 14 & Rt & Rt TLE plus $†$ & Rt ATL extended to Ins \\
\hline 13 & M & 61 & 40 & 21.4 & 6 & $\mathrm{~N}$ & 20 & Rt & Rt MTLE & Rt LiTT HG \\
\hline \multicolumn{11}{|l|}{ MED } \\
\hline 14 & $F$ & 43 & 39 & 3.9 & 4 & $\mathrm{~N}$ & 15 & $\mathrm{Lt}$ & Lt MTLE & $\begin{array}{l}\text { Scheduled for It HG } \\
\quad \text { RNS }\end{array}$ \\
\hline 15 & M & 30 & 11 & 18.6 & 8 & $\mathrm{EH}$ & 15 & $\mathrm{Lt}$ & Nonlocalized & $\begin{array}{l}\text { Offered DBS (failed } \\
\text { VNS) }\end{array}$ \\
\hline 16 & $\mathrm{~F}$ & 23 & 13 & 9.5 & 5 & $\mathrm{~N}$ & 8 & $\mathrm{Lt}$ & Lt MTLE & $\begin{array}{l}\text { Lt HG, subtemporal } \\
\quad \text { RNS }\end{array}$ \\
\hline 17 & $\mathrm{~F}$ & 40 & 14 & 25.7 & 7 & $\mathrm{~N}$ & 8 & Rt & Rt MTLE & Rt ATL \\
\hline 18 & $\mathrm{~F}$ & 42 & 8 & 34.0 & 12 & HS+ & 18 & Lt & Lt TLE plus, $\dagger$ rt MTLE & Palliative It HG LiTT \\
\hline 19 & M & 46 & 41 & 4.7 & 3 & $\mathrm{HS}$ & 16 & Rt & Rt TLE: Tp subtype & Rt ATL \\
\hline 20 & M & 27 & 13 & 13.6 & 5 & $\mathrm{HS}$ & 14 & $\mathrm{Lt}$ & Lt MTLE & Lt ATL \\
\hline 21 & M & 34 & 20 & 13.9 & 5 & $\mathrm{EH}$ & 15 & Rt & Rt lat TLE & Rt lat resection \\
\hline 22 & $\mathrm{~F}$ & 40 & 8 & 32.0 & 8 & $\mathrm{HS}$ & 16 & $\mathrm{Lt}$ & Lt TLE plus $†$ & $\begin{array}{l}\text { Scheduled palliative It } \\
\text { HG LiTT }\end{array}$ \\
\hline 23 & $M$ & 24 & 16 & 8.1 & 3 & $\mathrm{HS}+$ & 15 & Rt & Bilat TLE & Bilat HG RNS \\
\hline 24 & $\mathrm{~F}$ & 44 & 33 & 11.0 & 8 & $\mathrm{HS}+$ & 13 & $\mathrm{Lt}$ & Lt TLE plus† & $\begin{array}{l}\text { Scheduled palliative It } \\
\text { HG LiTT }\end{array}$ \\
\hline
\end{tabular}

ACing $\mathrm{G}$ = anterior cingulate gyrus; $\mathrm{AEDs}$ = antiepileptic drugs; $\mathrm{ATL}$ = anterior temporal lobectomy; $\mathrm{EH}$ = extrahippocampal pathology; $\mathrm{EZ}=$ epileptogenic zone; $\mathrm{HG}=$ hippocampal gyrus; HS = hippocampal sclerosis; HS+ = hippocampal sclerosis along with additional temporal lobe pathology; Ins = insula; LiTT = laser interstitial thermal therapy; $\mathrm{MTLE}=$ mesial TLE; $\mathrm{N}=$ normal; OperG = opercular gyrus; OrbF = orbitofrontal; $p t=$ patient; RNS = responsive neuronal stimulation; $\mathrm{Tp}=$ temporopolar;

VNS = vagal nerve stimulation

* Mesial to lateral temporal subtype of TLE.

† "TLE plus" indicates multiple seizure onset sites including the temporal lobe.

\section{Results}

\section{Patient Demographics}

Details regarding patient demographics are shown in Table 1. A total of 24 patients underwent thalamic SEEG implantation. The ANT was targeted in 13 patients
(54.2\%), and the MED group nuclei were targeted in 11 patients $(45.8 \%$ ) (male/female ratio 11:13, mean age at implantation $40 \pm 11$ years). Brain MR images were negative for any epileptogenic lesions in 10 patients $(41.7 \%)$, while 4 patients $(16.7 \%)$ had hippocampal sclerosis, 5 (20.8\%) 
A

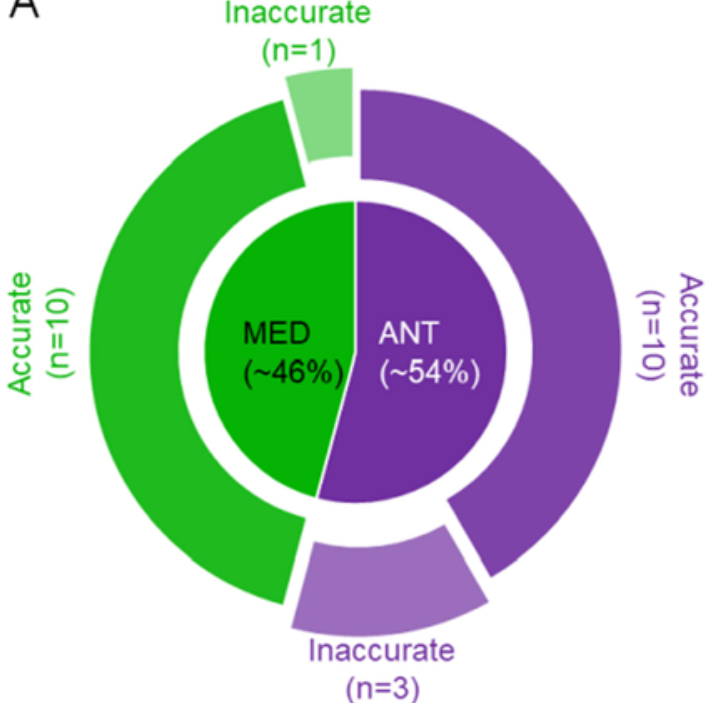

B

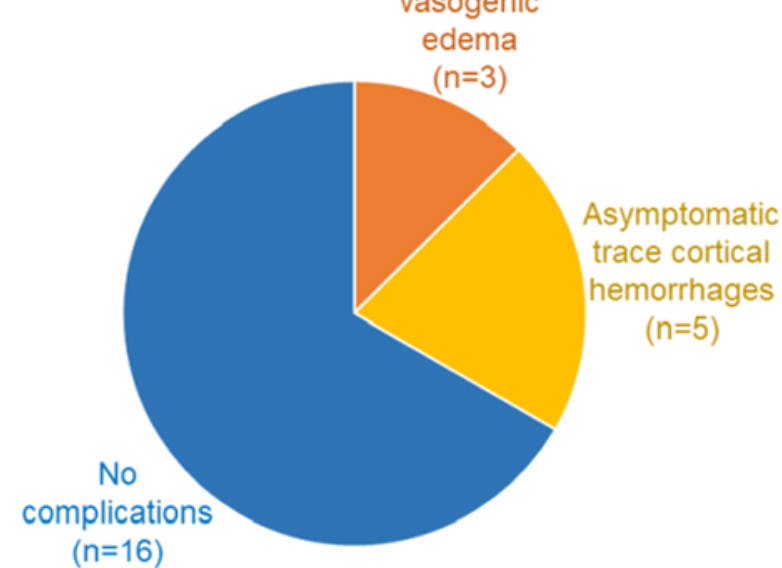

${ }^{* *}$ No hemorrhage or edema in thalamus
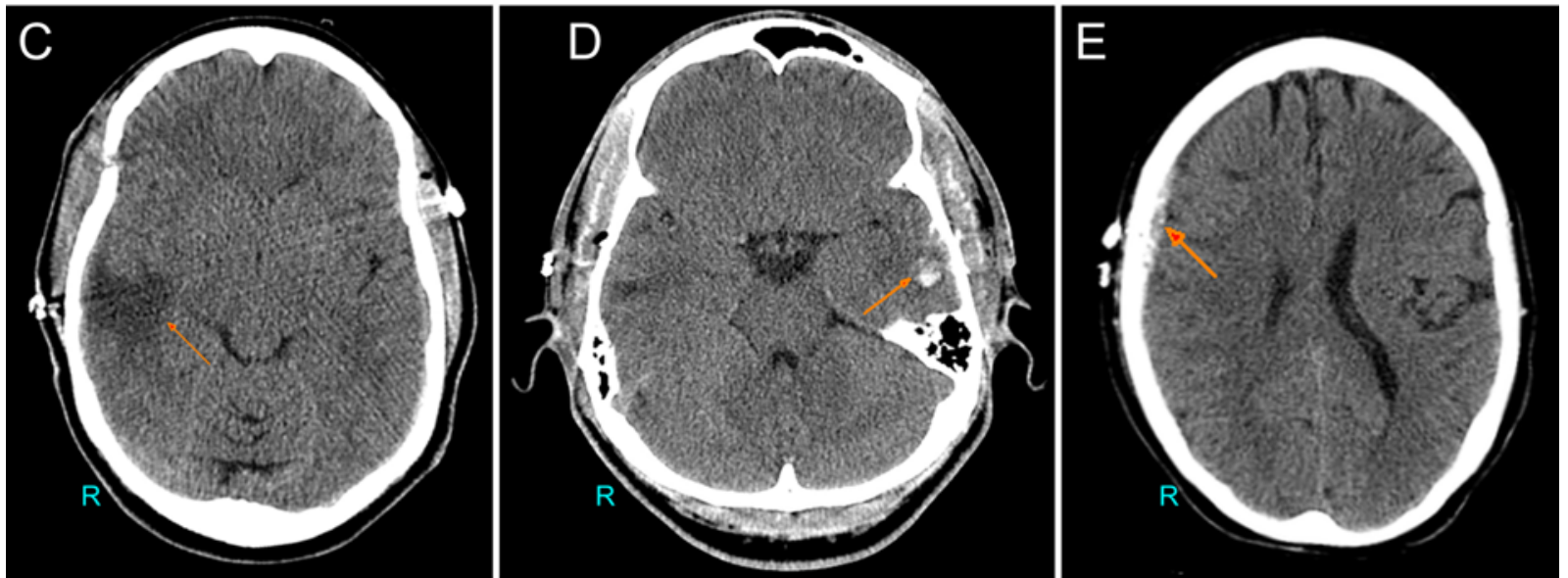

FIG. 4. Thalamic implant and complication rates. A: Pie chart showing the number of patients receiving ANT or MED thalamic implants. B: Pie chart demonstrating the number of patients who had postoperative complications in terms of asymptomatic vasogenic edema and asymptomatic hemorrhages in the proximity of the entry site of the electrodes. C: Postexplantation CT scan showing asymptomatic vasogenic edema (arrow) in the right temporal lobe. D: Postexplantation CT scan demonstrating an asymptomatic left temporal intracranial hematoma (arrow). E: Postexplantation CT scan showing an asymptomatic right frontal extradural hematoma (arrow).

had hippocampal sclerosis with additional extrahippocampal pathology, and the remaining 5 (20.8\%) had extrahippocampal pathology only.

\section{SEEG Implantation and Complications}

On average, each patient received a median of 172 contacts (range 102-274 contacts). SEEG implantation and outcome data are presented in Table 1. None of the patients had thalamic hemorrhage or edema. Asymptomatic subarachnoid, subdural, and intracranial hemorrhages were noted close to the entry site of the electrodes in $5(21 \%)$ of 24 patients (Fig. 4B). All hemorrhages were grade 1-2 according to McGovern's SEEG hemorrhage grading ${ }^{23}$ (consisting of a small intracranial bleed, either close to or away from eloquent cortex); these were low-grade hemorrhages with a lower probability of being symptomatic. On evaluating the 3 major risk factors as identified by McGovern et al., we found no significant difference in sex, age, or number of electrode implants for patients with and without hemorrhage (male/female ratio 4:1, $\mathrm{t}=3.8, \mathrm{p}=0.051$; age of patients in the hemorrhage group $42.7 \pm 7$ years and age of patients in the no-hemorrhage group $39.4 \pm 11$ years, $\mathrm{t}=0.58$, $\mathrm{p}=0.56$; number of electrodes in the hemorrhage group 14 \pm 4 and number of electrodes in the no-hemorrhage group $14 \pm 3, \mathrm{t}=0.34, \mathrm{p}=0.73$ ). Asymptomatic vasogenic edema in the temporal or parietal lobe was noted in 3 of the 24 patients. Overall, $34 \%$ of patients had asymptomatic hemorrhage or edema shown on the postexplantation CT scans. Follow-up CT scans showed resolution of these findings. None of the patients had any symptomatic hemorrhage or required any surgical interventions to treat hemorrhage.

\section{Targeting Accuracy}

Of the 13 patients who underwent planned ANT im- 
TABLE 2. Measurements of electrode targets to anatomical landmarks

\begin{tabular}{|c|c|c|c|c|c|c|c|c|c|c|c|c|}
\hline \multirow{3}{*}{ ANT } & \multicolumn{3}{|c|}{ Target (mm) } & \multicolumn{3}{|c|}{$\mathrm{FM}(\mathrm{mm})$} & \multicolumn{3}{|c|}{$\mathrm{MI}(\mathrm{mm})$} & \multicolumn{3}{|c|}{$\mathrm{TV}(\mathrm{mm})$} \\
\hline & $x$ & $\mathrm{Y}$ & Z & $x$ & Y & Z & $x$ & Y & Z & $x$ & Y & Z \\
\hline & & & & & & & & & & & & \\
\hline \multicolumn{13}{|l|}{ Location } \\
\hline $\mathrm{Lt}(\mathrm{n}=1)$ & -5 & -5 & 8 & -4 & 1 & 5 & 0 & -12 & 10 & 0 & -12 & -4 \\
\hline \multirow[t]{2}{*}{$\operatorname{Rt}(n=9)$} & $2 \pm 5$ & $-6 \pm 2$ & $6 \pm 7$ & $2 \pm 1$ & $0 \pm 1$ & $3 \pm 2$ & $0 \pm 0$ & $-11 \pm 2$ & $2 \pm 5$ & $0 \pm 0$ & $-12 \pm 1$ & $-4.1 \pm 1$ \\
\hline & \multicolumn{3}{|c|}{ Target to FM } & \multicolumn{3}{|c|}{ Target to MI } & \multicolumn{3}{|c|}{ Target to TV } & & & \\
\hline \multicolumn{13}{|c|}{ Linear component distance } \\
\hline Lt & 1 & 6 & 3 & 5 & 7 & 2 & 5 & 7 & 12 & & & \\
\hline \multirow[t]{2}{*}{ Rt } & $3 \pm 4$ & $6 \pm 2$ & $6 \pm 3$ & $5 \pm 2$ & $5 \pm 2$ & $7 \pm 5$ & $5 \pm 2$ & $7 \pm 2$ & $11 \pm 5$ & & & \\
\hline & to FM & to $\mathrm{Ml}$ & to TV & & & & & & & & & \\
\hline \multicolumn{13}{|c|}{ Euclidian distance } \\
\hline Lt & 7 & 9 & 15 & & & & & & & & & \\
\hline \multirow[t]{3}{*}{$\mathrm{Rt}$} & $10 \pm 3$ & $11 \pm 3$ & $15 \pm 2$ & & & & & & & & & \\
\hline & \multicolumn{3}{|c|}{ Target $(\mathrm{mm})$} & \multicolumn{3}{|c|}{$\mathrm{FM}(\mathrm{mm})$} & \multicolumn{3}{|c|}{$\mathrm{Ml}(\mathrm{mm})$} & \multicolumn{3}{|c|}{ TV $(\mathrm{mm})$} \\
\hline & $x$ & $Y$ & Z & $X$ & $Y$ & Z & $x$ & $Y$ & Z & $X$ & $Y$ & Z \\
\hline \multicolumn{13}{|l|}{ MED } \\
\hline \multicolumn{13}{|l|}{ Location } \\
\hline Lt $(n=8)$ & $-3 \pm 1$ & $-10 \pm 3$ & $2 \pm 4$ & $-3 \pm 1$ & $-1 \pm 1$ & $2 \pm 2$ & $0 \pm 0$ & $-10 \pm 2$ & $2 \pm 5$ & $0 \pm 0$ & $-12 \pm 1$ & $-4 \pm 1$ \\
\hline \multirow[t]{2}{*}{$\operatorname{Rt}(n=2)$} & $5 \pm 3$ & $-12 \pm 1$ & $1 \pm 4$ & $2 \pm 1$ & $0 \pm 2$ & $3 \pm 4$ & $0 \pm 0$ & $-10 \pm 1$ & $-2 \pm 1$ & $0 \pm 0$ & $-13 \pm 2$ & -51 \\
\hline & \multicolumn{3}{|c|}{ Target to FM } & \multicolumn{3}{|c|}{ Target to Ml } & \multicolumn{3}{|c|}{ Target to TV } & & & \\
\hline \multicolumn{13}{|c|}{ Linear component distance } \\
\hline Lt & $0 \pm 1$ & $9 \pm 3$ & $4 \pm 2$ & $2 \pm 1$ & $2 \pm 3$ & $2 \pm 3$ & $2 \pm 1$ & $3 \pm 3$ & $7 \pm 3$ & & & \\
\hline \multirow[t]{2}{*}{ Rt } & $4 \pm 3$ & $12 \pm 2$ & $3 \pm 2$ & $5 \pm 3$ & $2 \pm 1$ & $3 \pm 3$ & $5 \pm 3$ & $1 \pm 2$ & $6 \pm 5$ & & & \\
\hline & to FM & to $\mathrm{Ml}$ & to TV & & & & & & & & & \\
\hline \multicolumn{13}{|c|}{ Euclidian distance } \\
\hline Lt & $10 \pm 2$ & $5 \pm 3$ & $8 \pm 4$ & & & & & & & & & \\
\hline Rt & $13 \pm 2$ & $7 \pm 2$ & $9 \pm 3$ & & & & & & & & & \\
\hline
\end{tabular}

FM = foramen of Monro; $\mathrm{MI}=$ massa intermedia; TV = anteroposterior midpoint of third ventricle.

The measurements (in $\mathrm{mm}$ ) between the electrode target and the anatomical landmarks are provided in terms of actual 3D coordinates of the electrodes, their linear components vectorized on the $x, y$, and $z$ axes, and, finally, the 3D euclidean distance in space between the target tip and the anatomical landmark. Values are presented as mean $\pm \mathrm{SD}$.

plantation, $10(77 \%)$ had confirmed localization in the ANT (Fig. 4A). In one of the patients, the electrode passed through the ipsilateral ANT and crossed the midline to the contralateral thalamus, traversing through the mediodorsal nucleus. In another patient, the electrode target stopped short by $4 \mathrm{~mm}$ and was situated in the ventral lateral nucleus of the thalamus. In the third patient, the electrode target was situated $3 \mathrm{~mm}$ anterior to the ANT in the anterior fornix.

For the 11 patients who underwent MED implantation, the massa intermedia was used for localization. Ten electrodes $(91 \%)$ were situated in the MED (CeM and mediodorsal). In one patient, the electrode target stopped short by $8 \mathrm{~mm}$ from the midline and was situated in the ventromedial thalamic nucleus.

Postimplantation accuracies on the $\mathrm{x}, \mathrm{y}$, and $\mathrm{z}$ axes and euclidean errors are presented in Table 2. The trajectories for the ANT group differed slightly from those of the
MED group at the entry point, which was in the precentral gyrus in the former and in the postcentral gyrus in the latter (Fig. 5).

Once the implantation process was completed, SEEG data were recorded continuously. The thalamic SEEG signals in all patients were interpretable and comparable to those of the cortical channels. However, the local field potentials of the thalamus were of a lower amplitude than those of the cortical channels (Fig. 6). The electrophysiological data thus obtained were analyzed and published previously, addressing key clinical questions. ${ }^{26,27,29,40}$

\section{Discussion}

Stereotactic procedures targeting the thalamus date back to the mid-20th century when Spiegel and Wycis reported on thalamotomy for several psychiatric indications ${ }^{33}$ and these authors were also the first to record sei- 

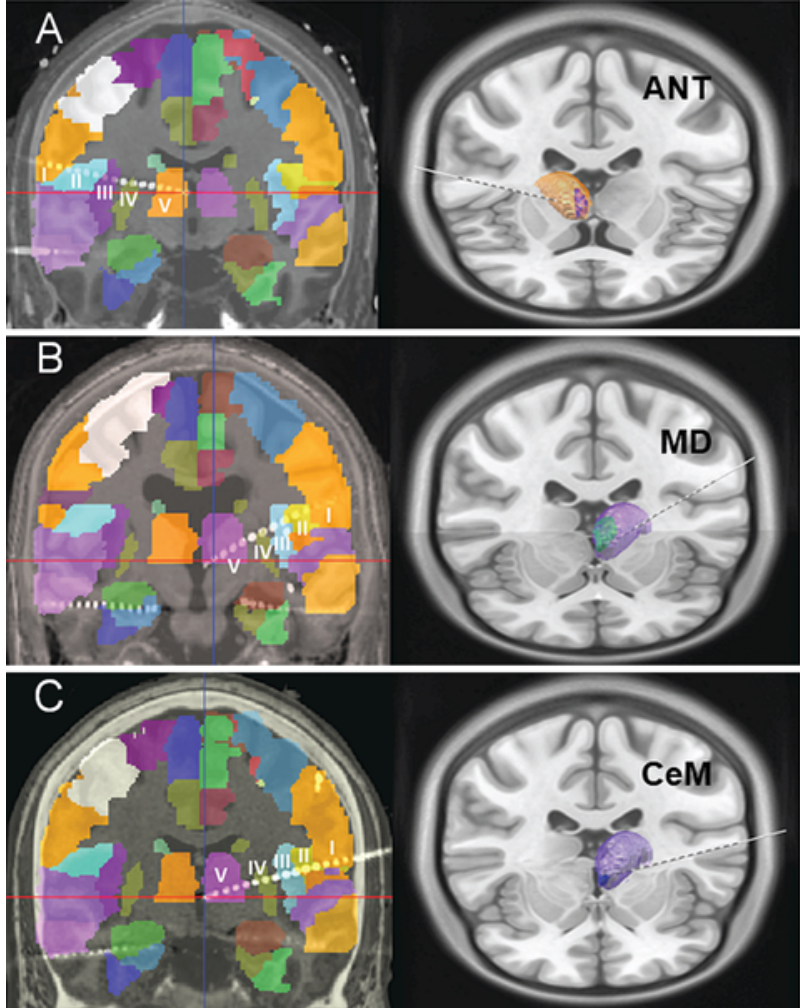

I - precentral gyrus (PreCG) II - pars opercularis frontalis III - Insula IV - Putamen $\mathrm{V}$ - Thalamus

FIG. 5. Implant registration accuracy: Lead-DBS was used to reconstruct the target location of the thalamic electrodes, while the trajectories were reconstructed, and the cortical contacts were identified using iElectrodes. A: ANT implantation followed the trajectory of the precentral gyrus, pars opercularis frontalis, insula, putamen, and thalamus. B and C: MED group implantation followed a similar trajectory except that the entry point was the postcentral gyrus. D and E: The group implants showing the trajectories of all ANT and MED group implants, respectively.

zure activity from this location. ${ }^{38}$ Since then, numerous thalamic stereotactic procedures have been performed for a wide variety of indications. However, by far the most significant experience in thalamic stereotaxy has been the implantation of deep brain stimulation (DBS) electrodes in the ventral intermediate thalamus for the management of essential tremor. The published complication rates of these DBS procedures mirror those of SEEG overall (range 1\%1.3\%). ${ }^{8,11,14}$ In one pivotal study, McGovern et al. reported an overall hemorrhage rate of $19.1 \%,{ }^{23}$ while in our study the hemorrhage rate was $20.8 \%$, with all hemorrhages occurring at the entry site of the electrodes. Contrary to the common belief that thalamic implantation is associated with a high bleed rate, in our study we noted no thalamic bleeds. In the pivotal trial for ANT DBS, $4.5 \%$ of the 110 patients had incidental asymptomatic intracerebral hemorrhage, but it is unclear if these bleeds were around the entry site of the DBS electrodes or if they were thalamic. ${ }^{13,34}$ Overall, SEEG requires an increased number of brain penetrations compared to DBS; however, the electrodes are smaller, and the procedure is very well tolerated, with a reported hemorrhage rate of $1 \%-4 \% .^{8,11,14,24}$ The higher complication rate reported in the study may be due to differences in reporting. Prior SEEG studies have reported hemorrhage rates based on evaluating postimplantation brain CT scans, while in the present study, postexplantation CT scans were used to estimate complications, similar to what was done by McGovern et al. An electrophysiological sampling of the thalamus during SEEG investigation has been reported, but complications were not studied in detail.1,12,15,30,31

Although the thalamus is successfully and safely targeted in DBS, SEEG requires quite different trajectory planning and implantation techniques. In contrast to DBS, where there is considerable flexibility in choosing the entry point, SEEG entry points and the target, as well as the structures along the path to the target, are frequently constrained by MRI abnormalities, magnetoencephalography or PET findings, intervening sulci, and vasculature. Also, thalamic DBS affords the opportunity, in many cases, to fully visualize the cortex if the dura is opened fully, whereas SEEG electrodes are placed via a small craniostomy in which direct visualization is not possible. Furthermore, a rigid cannula is passed either to the target or just shy of the target to guide placement of DBS electrodes, whereas SEEG electrodes are placed without a cannula and are more prone to deviations. Finally, SEEG surgical planning requires achieving adequate coverage of the putative epileptogenic areas with a limited number of electrodes. In suspected TLE, anatomical sampling with SEEG often includes extratemporal structures-including the insular operculum and the orbitofrontal, parietal, and cingulate regions-to rule out a lesion mimicking TLE. ${ }^{2,20}$ Numerous stereotactic techniques have been described for depth 


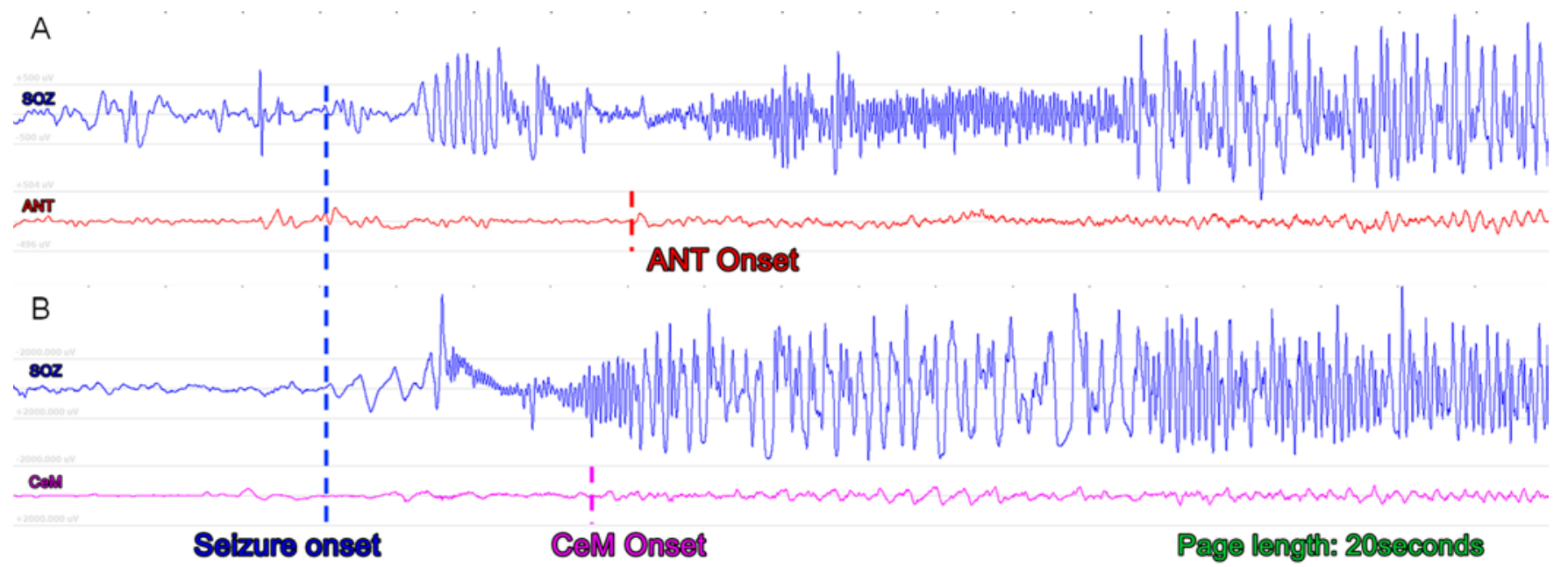

FIG. 6. Seizure recording from the thalamus. A depiction of seizures recorded in the thalamus. The identification of the unequivocal electrographic onset was done by the clinician in the seizure onset channels (SOZ). Subsequent review of the thalamic channel showed that shortly after the seizure was noted in the SOZ, there was an evolving ictal rhythm noted in the thalamic channels. This EEG activity change was of a lower amplitude compared to that of the cortical channels and had a different morphology. A: An evolving ictal rhythm in a thalamic channel recording from ANT during a focal seizure originating in the ipsilateral hippocampus. B: Similar ictal activity recorded from the CeM nucleus during a focal seizure originating from the ipsilateral hippocampus.

electrode placement, including frame-based, frameless, and robotic methods, each with its own relative advantages and disadvantages. Many surgeons have recently gravitated toward robotic methods due to the precision and speed offered. We do not endorse any particular technique; in fact, we believe thalamic depth electrode implantation is likely safe when using any modern stereotactic system that has an accuracy error of approximately $1 \mathrm{~mm}$ or less. We maintain that safety is more a factor of careful trajectory planning, taking care to avoid cortical, sulcal, or deep vasculature and to ensure accurate image registration and cautious surgical technique that avoids common complications, such as drill skiving, drill plunging, and human measurement errors. These fundamentals are critical for the safety of all stereotactic procedures. We utilized a single technique and therefore cannot directly address the safety or accuracy differences of the various stereotactic methods.

Despite these myriad technical constraints, here our results demonstrate that it is safe to extend clinically indicated trajectories, specifically those through the frontal operculum or insula, for accurate targeting of the limbic thalamus. The overall complication rates are low and comparable to those of electrodes placed in any other location. With these results, we propose that if patients are fully informed of the risks involved, there are significant benefits to obtaining robust signals from thalamic nuclei involved in seizure networks, which may help guide future therapies. ${ }^{26,27,29}$ Some of the early studies by our group have shown the following: 1) periictal electrophysiological changes occurring in the thalamus during the seizures, 2) cortical responsiveness to thalamic stimulation, and 3) a temporal predictive model to determine ictal and interictal thalamic states in TLE. In concordance with the growing evidence from various centers around the world, there is a possibility to envisage a more patient-oriented closed-loop
DBS system. Systematic evaluation of complex thalamocortical interactions will eventually help in the development of such neuromodulation interventions in patients with drug-resistant epilepsies. Current thalamic DBS strategies are based mostly on a one-size-fits-all model without knowledge of the thalamocortical interactions specific to a given patient. Estimating patient-specific inherent thalamocortical frequency interactions can help in tailoring the stimulation parameters and developing DBS systems to optimize clinical response, which could significantly improve their clinical outcomes.

\section{Limitations}

From our collective experience, we highlight some of the challenges and future perspectives about thalamic sampling during SEEG investigation. First, target selection was performed directly on a $3 \mathrm{~T}$ standard T1-weighted gadolinium-enhanced MRI sequence, which is challenging since both the ANT and MED are not well visualized. While used as an external visual reference, Morel's thalamic atlas overlay or its coordinate system ${ }^{21}$ has not, to date, been integrated into the robotic navigation systems, and we did not use specialized MRI sequences to visualize landmarks such as the mammillothalamic tract on an FGATIR (fast gray matter acquisition T1 inversion recovery) sequence. With experience and with potential incorporation of deformable atlases, we anticipate that our targeting process will become more precise. Second, although no hemorrhagic or focal neurological complications were noted, detailed neuropsychiatric examinations were not performed to assess whether the routine placement of electrodes produces damage that results in cognitive decline. ${ }^{42}$ A randomized trial of ANT DBS did not find any cognitive decline associated with the placement of the ANT DBS system. However, the transventricular 
trajectory utilized in that study is different from the lateral trajectory used in the current study, making direct comparison difficult. Variable neuropsychological changes have been reported following ventral intermediate DBS that, when present, are thought to be primarily stimulation related and not the result of a lesion. ${ }^{22,45}$

\section{Conclusions}

The therapeutic potential and prognostic role of the thalamus in focal epilepsy are well established in preclinical and clinical imaging studies. However, the lack of electrophysiological studies limits our knowledge of its involvement and may potentially hinder the development of therapies. Using robot-assisted SEEG, we have demonstrated the safety of electrophysiological sampling from various thalamic nuclei for research recordings and have presented a technique that avoids implanting additional depth electrodes or compromising clinical care. We state with utmost caution that the current results should not be mistaken for a safety blanket; instead, safe deep brain structure implantation should be judiciously performed only after the development of meticulous anatomical target strategies and robotic planning to avoid untoward complications during the SEEG procedure.

\section{Acknowledgments}

We would like to acknowledge the contribution of patients and their family members without whom this study would be incomplete. Ms. Jennifer Mahaffey and Ms. Cynthia Stover, Department of Neurology, University of Alabama at Birmingham (UAB), have extensively coordinated and organized the initial administrative components of the study. We would also like to recognize the continued enthusiasm and support from the EEG technicians at the UAB Epilepsy Center. S.P. and G.C. would like to acknowledge support from NIH (1RF1MH117155-01), and S.P. and A. Irannejad would like to acknowledge support from the EPSCoR program at the National Science Foundation (NSF RII-2FEC OIA1632891)

\section{References}

1. Arthuis M, Valton L, Régis J, Chauvel P, Wendling F, Naccache L, et al: Impaired consciousness during temporal lobe seizures is related to increased long-distance cortical-subcortical synchronization. Brain 132:2091-2101, 2009

2. Aupy J, Noviawaty I, Krishnan B, Suwankpakdee P, Bulacio $\mathrm{J}$, Gonzalez-Martinez J, et al: Insulo-opercular cortex generates oroalimentary automatisms in temporal seizures. Epilepsia 59:583-594, 2018

3. Avants BB, Tustison N, Song G: Advanced Normalization Tools (ANTs). Insight J 2:1-35, 2009

4. Barba C, Rheims S, Minotti L, Guénot M, Hoffmann D, Chabardès $\mathrm{S}$, et al: Temporal plus epilepsy is a major determinant of temporal lobe surgery failures. Brain 139:444451, 2016

5. Baydin S, Gungor A, Baran O, Tanriover N, Rhoton AL: The double massa intermedia. Surg Neurol Int 7:30, 2016

6. Bertram EH, Zhang D, Williamson JM: Multiple roles of midline dorsal thalamic nuclei in induction and spread of limbic seizures. Epilepsia 49:256-268, 2008

7. Blenkmann AO, Phillips HN, Princich JP, Rowe JB, Bekinschtein TA, Muravchik CH, et al: iElectrodes: a comprehensive open-source toolbox for depth and subdural grid electrode localization. Front Neuroinform 11:14, 2017
8. Cardinale F, Cossu M, Castana L, Casaceli G, Schiariti MP, Miserocchi A, et al: Stereoelectroencephalography: surgical methodology, safety, and stereotactic application accuracy in 500 procedures. Neurosurgery 72:353-366, 2013

9. Cassidy RM, Gale K: Mediodorsal thalamus plays a critical role in the development of limbic motor seizures. J Neurosci 18:9002-9009, 1998

10. Chabardès S, Kahane P, Minotti L, Tassi L, Grand S, Hoffmann $\mathrm{D}$, et al: The temporopolar cortex plays a pivotal role in temporal lobe seizures. Brain 128: 1818-1831, 2005

11. Cossu M, Cardinale F, Castana L, Citterio A, Francione S, Tassi L, et al: Stereoelectroencephalography in the presurgical evaluation of focal epilepsy: a retrospective analysis of 215 procedures. Neurosurgery 57:706-718, 2005

12. Evangelista E, Bénar C, Bonini F, Carron R, Colombet B, Régis J, et al: Does the thalamo-cortical synchrony play a role in seizure termination? Front Neurol 6:192, 2015

13. Fisher R, Salanova V, Witt T, Worth R, Henry T, Gross R, et al: Electrical stimulation of the anterior nucleus of thalamus for treatment of refractory epilepsy. Epilepsia 51:899-908, 2010

14. González-Martínez J, Bulacio J, Thompson S, Gale J, Smithason S, Najm I, et al: Technique, results, and complications related to robot-assisted stereoelectroencephalography. Neurosurgery 78:169-180, 2016

15. Guye M, Régis J, Tamura M, Wendling F, McGonigal A, Chauvel P, et al: The role of corticothalamic coupling in human temporal lobe epilepsy. Brain 129:1917-1928, 2006

16. Hamani C, Ewerton FI, Bonilha SM, Ballester G, Mello LE, Lozano AM: Bilateral anterior thalamic nucleus lesions and high-frequency stimulation are protective against pilocarpine-induced seizures and status epilepticus. Neurosurgery 54:191-197, 2004

17. He X, Doucet GE, Pustina D, Sperling MR, Sharan AD, Tracy JI: Presurgical thalamic "hubness" predicts surgical outcome in temporal lobe epilepsy. Neurology 88:2285-2293, 2017

18. Horn A, Li N, Dembek TA, Kappel A, Boulay C, Ewert S, et al: Lead-DBS v2: Towards a comprehensive pipeline for deep brain stimulation imaging. Neuroimage 184:293-316, 2019

19. Isnard J, Guénot M, Ostrowsky K, Sindou M, Mauguière F: The role of the insular cortex in temporal lobe epilepsy. Ann Neurol 48: 614-623, 2000

20. Kahane P, Barba C, Rheims S, Job-Chapron AS, Minotti L, Ryvlin P: The concept of temporal 'plus' epilepsy. Rev Neurol (Paris) 171:267-272, 2015

21. Krauth A, Blanc R, Poveda A, Jeanmonod D, Morel A, Székely G: A mean three-dimensional atlas of the human thalamus: generation from multiple histological data. Neuroimage 49:2053-2062, 2010

22. Loher TJ, Gutbrod K, Fravi NL, Pohle T, Burgunder JM, Krauss JK: Thalamic stimulation for tremor. Subtle changes in episodic memory are related to stimulation per se and not to a microthalamotomy effect. J Neurol 250:707-713, 2003

23. McGovern RA, Ruggieri P, Bulacio J, Najm I, Bingaman WE, Gonzalez-Martinez JA: Risk analysis of hemorrhage in stereo-electroencephalography procedures. Epilepsia 60:571-580, 2019

24. Mullin JP, Shriver M, Alomar S, Najm I, Bulacio J, Chauvel $\mathrm{P}$, et al: Is SEEG safe? A systematic review and meta-analysis of stereo-electroencephalography-related complications. Epilepsia 57:386-401, 2016

25. Paz JT, Davidson TJ, Frechette ES, Delord B, Parada I, Peng $\mathrm{K}$, et al: Closed-loop optogenetic control of thalamus as a tool for interrupting seizures after cortical injury. Nat Neurosci 16:64-70, 2013

26. Pizarro D, Ilyas A, Chaitanya G, Toth E, Irannejad A, Romeo A, et al: Spectral organization of focal seizures within the thalamotemporal network. Ann Clin Transl Neurol 6:18361848,2019 
27. Pizarro D, Ilyas A, Toth E, Romeo A, Riley KO, Esteller R, et al: Automated detection of mesial temporal and temporoperisylvian seizures in the anterior thalamic nucleus. Epilepsy Res 146:17-20, 2018

28. Rolls ET, Joliot M, Tzourio-Mazoyer N: Implementation of a new parcellation of the orbitofrontal cortex in the automated anatomical labeling atlas. Neuroimage 122:1-5, 2015

29. Romeo A, Issa Roach AT, Toth E, Chaitanya G, Ilyas A, Riley KO, et al: Early ictal recruitment of midline thalamus in mesial temporal lobe epilepsy. Ann Clin Transl Neurol 6:1552-1558, 2019

30. Rosenberg DS, Mauguière F, Catenoix H, Faillenot I, Magnin M: Reciprocal thalamocortical connectivity of the medial pulvinar: a depth stimulation and evoked potential study in human brain. Cereb Cortex 19:1462-1473, 2009

31. Rosenberg DS, Mauguière F, Demarquay G, Ryvlin P, Isnard $\mathrm{J}$, Fischer C, et al: Involvement of medial pulvinar thalamic nucleus in human temporal lobe seizures. Epilepsia 47:98 107, 2006

32. Ryvlin P, Kahane P: The hidden causes of surgery-resistant temporal lobe epilepsy: extratemporal or temporal plus? Curr Opin Neurol 18:125-127, 2005

33. Rzesnitzek L, Hariz M, Krauss JK: The origins of human functional stereotaxis: a reappraisal. Stereotact Funct Neurosurg 97:49-54, 2019

34. Salanova V, Witt T, Worth R, Henry TR, Gross RE, Nazzaro $\mathrm{JM}$, et al: Long-term efficacy and safety of thalamic stimulation for drug-resistant partial epilepsy. Neurology 84:10171025,2015

35. Schönecker T, Kupsch A, Kühn AA, Schneider GH, Hoffmann KT: Automated optimization of subcortical cerebral MR imaging-atlas coregistration for improved postoperative electrode localization in deep brain stimulation. AJNR Am J Neuroradiol 30:1914-1921, 2009

36. Sherdil A, Coizet V, Pernet-Gallay K, David O, Chabardès S, Piallat B: Implication of anterior nucleus of the thalamus in mesial temporal lobe seizures. Neuroscience 418:279-290, 2019

37. Sloan DM, Bertram EH III: Changes in midline thalamic recruiting responses in the prefrontal cortex of the rat during the development of chronic limbic seizures. Epilepsia 50:556-565, 2009

38. Spiegel E, Wycis H: Thalamic recordings in man with special reference to seizure discharges. Electroencephalogr Clin Neurophysiol 2:23-27, 1950

39. Thom M, Mathern GW, Cross JH, Bertram EH: Mesial temporal lobe epilepsy: how do we improve surgical outcome? Ann Neurol 68:424-434, 2010
40. Toth E, Chaitanya G, Pati S: Mapping short-latency cortical responses to electrical stimulation of thalamic motor nuclei by increasing sampling rate-a technical report. Clin Neurophysiol 131:142-144, 2020

41. Tóth E, Fabó D, Entz L, Ulbert I, Erőss L: Intracranial neuronal ensemble recordings and analysis in epilepsy. J Neurosci Methods 260:261-269, 2016

42. Tröster AI, Meador KJ, Irwin CP, Fisher RS, SANTE Study: Memory and mood outcomes after anterior thalamic stimulation for refractory partial epilepsy. Seizure 45:133-141, 2017

43. Van Gompel JJ, Stead SM, Giannini C, Meyer FB, Marsh WR, Fountain T, et al: Phase I trial: safety and feasibility of intracranial electroencephalography using hybrid subdural electrodes containing macro- and microelectrode arrays. Neurosurg Focus 25(3):E23, 2008

44. Wiebe S, Blume WT, Girvin JP, Eliasziw M: A randomized, controlled trial of surgery for temporal-lobe epilepsy. N Engl J Med 345:311-318, 2001

45. Woods SP, Fields JA, Lyons KE, Koller WC, Wilkinson SB, Pahwa R, et al: Neuropsychological and quality of life changes following unilateral thalamic deep brain stimulation in Parkinson's disease: a one-year follow-up. Acta Neurochir (Wien) 143:1273-1278, 2001

\section{Disclosures}

The authors report no conflict of interest concerning the materials or methods used in this study or the findings specified in this paper.

\section{Author Contributions}

Conception and design: Pati, Chaitanya, Romeo, Riley. Acquisition of data: Pati, Romeo, Ilyas, Irannejad, Elsayed, Bentley, Riley. Analysis and interpretation of data: Pati, Chaitanya, Irannejad, Toth. Drafting the article: Pati, Chaitanya, Romeo, Ilyas. Critically revising the article: Pati, Chaitanya, Romeo, Bentley, Riley. Reviewed submitted version of manuscript: Chaitanya, Toth. Approved the final version of the manuscript on behalf of all authors: Pati. Study supervision: Pati.

\section{Correspondence}

Sandipan Pati: Cognitive Neurophysiology Laboratory, University of Alabama at Birmingham, AL. spati@uabmc.edu. 